\title{
A Flexible Endoscopic Sensing Module for Force Haptic Feedback Integration
}

\author{
Hamid Naghibi \\ Robotics and Mechatronics lab. \\ University of Twente \\ Enschede, The Netherlands \\ h.naghibibeidokhti@utwente.nl
}

\author{
Willem B. Hoitzing \\ Robotics and Mechatronics lab. \\ University of Twente \\ Enschede, The Netherlands \\ w.b.hoitzing@student.utwente.nl
}

\author{
Stefano Stramigioli \\ Robotics and Mechatronics lab. \\ University of Twente \\ Enschede, The Netherlands \\ s.stramigioli@utwente.nl
}

\author{
Momen Abayazid \\ Robotics and Mechatronics lab. \\ University of Twente \\ Enschede, The Netherlands \\ m.abayazid@utwente.nl
}

\begin{abstract}
Proper haptic feedback is a key aspect to the safety of a teleoperated endoscopic surgical robotics system. In this study we developed a flexible sensing module to measure the directional force, and a haptic control of a soft robotic endoscopic segment. The force amplitude and direction detection was based on the voltage changes in four strain gauges positioned at four quarters of a flexible cylinder. The flexible structure of the cylinder allowed for large deformation which can reduce the risk of tissue damage at interfaces. Inverse finite element analysis and finite element simulations were implemented to further improve and verify the developed system. The system including the haptic feedback controller could enhance operator navigation.
\end{abstract}

Keywords: Soft Robotics, Endoscope, Haptic Feedback, Force sensing, Finite Element Analysis

\section{INTRODUCTION}

Minimally invasive endoscopic surgery with the least incision in patient's body is gradually replacing purely open surgery, which reduces patient trauma and recovery duration. In minimally invasive endoscopic surgery the surgeon performs the surgery by means of either conventional endoscope (rigid or soft endoscope) or remotely controlled robot-assisted endoscope (teleoperated surgery). One of the main challenges in such endoscopic surgeries, is the lack of sensation while pushing the endoscope inside the body and also while performing the operation.

Proper haptic feedback is a key aspect to the safety of a teleoperated surgical robotics system. Direct interaction between instrument and patient is no longer sensed by the surgeon due to the remote-control nature of the system. Haptic feedback can supply the surgeon with cues about the endeffector's motion, giving them some insight into their actions.

The haptic feedback systems can be either based on kinematics feedback or force feedback. Previously a few kinematics feedback-based system were developed, as reviewed by Van der Meijden et al. [1]. For instance, Mura et al. (2016) developed a vision based haptic feedback for endoscopic application [2]. Reilink et al. (2011) also presented a haptic control system based on the endoscopic images [3]. In all of the kinematics-based systems, however, the position of the endoscope needs to be tracked which makes imaging modalities implementation or system complications unavoidable. Moreover, the forces may not be fullt representative of the real forces as the kinematics data are converted to the force feedback. This can increase the risk of tissue damaging as conversion of kinematics to force is not always possible in the complicated dynamic nonhomogeneous tissue-endoscope interactions.

The force feedback-based haptic, therefore, can be a more realistic indication of the forces during the surgery. Previously, several force sensing modules were developed to measure the force during the rigid endoscopy (e.g. [4]-[6]). As reviewed by Baldwin, only a few studies developed force feedback-based haptic system for soft endoscope [7]. Most of the designs were, however, meant for tendon-sheath endoscopes. With the increasing trend toward soft robotics in endoscopic applications, a haptic feedback system suitable for soft robotics endoscope is still missing. The aim of this study was therefore to develop a flexible sensing module to measure the directional force, and to develop a haptic control of a soft robotic endoscopic module.

\section{A. Design Principles}

\section{MATERIALS AND METHODS}

The sensing module needs to be soft and flexible to prevent damage to the surrounding tissues. It also needs to enable small force detection to guide the surgeon before the endoscope looping problem occurs. The sensing module should also be capable of navigating the user by measuring the amount and direction of the force. It should not limit the maneuverability of the endoscope by adding physical and structural constrains to the system. Besides, the module should not hinder the endoscopic instrumentations and camera view field.

\section{B. Experimental set-up}

A simple flexible rubber cylinder with external radius of $23.2 \mathrm{~mm}$ and height of $30 \mathrm{~mm}$ was fabricated using $3 \mathrm{D}$ printing technique. As schematically illustrated in Figure 1, Four strain gauges were attached using flexible glue, inside the cylindrical wall with four equally spaced angular distance $(0,90,180$ and $270^{\circ}$ ). Every individual strain gauge was integrated into a Wheatstone bridge that was balanced for a neutral state of the sensor (Figure 2-b). The outputs of the Wheatstone bridges were amplified to be able to measure the small signals with an Arduino Uno, which requires analog inputs within the range of $0-5 \mathrm{~V}$. The amplification circuitry used a rail-to-rail differential amplifier of the LTC1992 product family, with a second order low-pass filter (cut-off frequency:100 Hz), to achieve an amplification gain of 250 . 


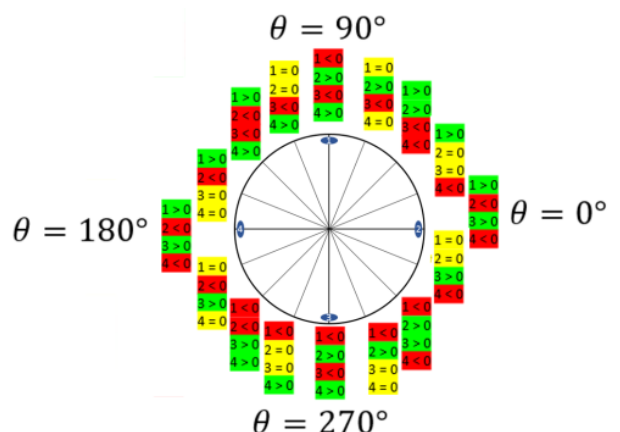

$$
\theta=270^{\circ}
$$

Fig. 1. Schematical illustration of the sensing module with strain gauges arrangements.

In order to calibrate the voltage change measurement from the strain gauges against the amplitude and orientation of external loads, a specific calibration set-up was designed (Figure 2). The calibration set-up allowed for applying different external forces (weights) in different orientation with angular step of $22.5^{\circ}$. four different weights $(62.6,80.6,112.8$ and $167.5 \mathrm{gr}$ ) were applied each in 12 equally spaced angles around the cylinder (with angular increment of $22.5^{\circ}$ ). during the experiments the voltage changes in all four strain gauges were recorded. Each loading condition was repeated five times to check the repeatability of the measurements.

\section{Verification of the set-up}

Finite element model of the flexible cylindrical module was developed in Abaqus (Simulia, Providence, RI). Neo-Hookean hyper-elastic material model was used to model the behavior of the cylinder. The material parameters were estimated based on the material data-sheet. The coefficients were further adjusted implementing inverse finite element analysis. For this purpose, three different radial forces were applied to the side of the cylindrical module wall and maximum corresponding deformations of the wall were measured. The material coefficients in FE simulation were adjusted to achieve similar deformation as measured in the experiment $(\mathrm{C} 10=0.27 \mathrm{MPa}$ and $\mathrm{D}=0.001)$. Similar to the experiment, all the described calibration loading conditions were applied to the FE model. In each simulation, the strain (the first principle logarithmic strain) at the nodes, where the strain gauges were positioned, was calculated. The strain corresponding to the experimentally measured voltage could be consequently determined. Moreover, the trend in the strain calculation (from the FE model) and voltage measurement (from the experiment) can be compared to verify the experimental measurements.

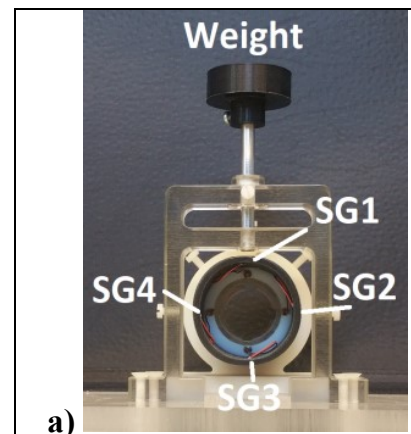

b)

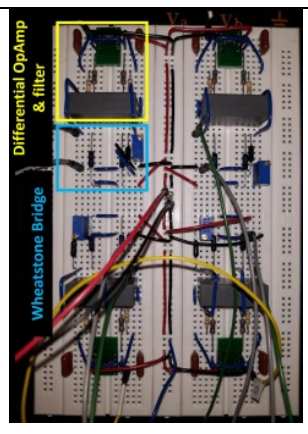

Fig. 2. The calibration set-up (a), and the electrical components configration of the set-up (b)

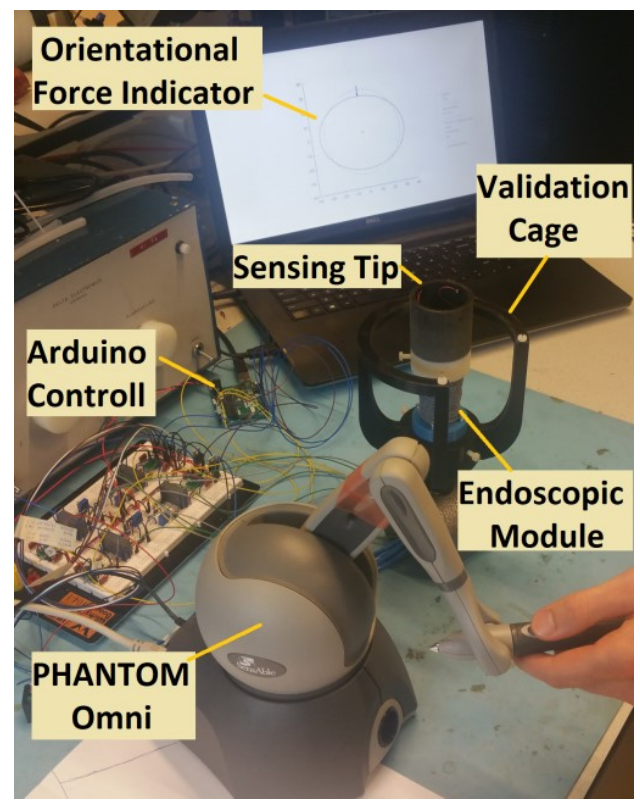

Fig. 3. The system Validation set-up

\section{Haptic feedback integration}

In order to assess the function of the force sensing cylindrical module in haptic feedback control, the cylindrical module was attached to a pneumatic soft endoscopic segment. The endoscopic segment can be bent in all four directions by pressurizing one of the four side chambers. The amount of bending at each direction can be achieved by controlling the pressure amplitude using the specification curve (input pressure vs. output bending) data. The directional bending of the endoscopic set-up including the sensing module can be controlled using a PHANTOM Omni which is a six-DOF haptic device capable of providing 3 DOF force feedback. The integration of the PHANTOM Omni requires communication of control parameters from the Omni to the soft actuators, as well as communication of environmental forces from the sensing module to the Omni. Both interactions between the Omni and the setup were interfaced using an Arduino Uno controlled using MATLAB. The end-effector of the Omni can be moved freely by the user, and the projections of the endeffector's position on a horizontal 2D plane are extracted and subsequently transferred to the pressure control of the soft actuator. The second interaction (the communication of environmental forces) are implemented straight-forward: the forces measured by the sensing module are parameterized into a magnitude and an angle relative to a pre-defined reference configuration of the sensing module. These parameters are sent to the Omni, which will then provide force feedback to the user. The force exerted on the user is in the same 2D plane as the user uses for the control of the actuator, and of appropriate magnitude and direction, mimicking the external force felt by the sensing module. 


\section{E. System Validation}

In order to verify if the user can be guided through the developed haptic feedback system, a plastic cage with an opening was modelled and 3D printed. The endoscopic unit (with the sensing module) was positioned at the center of the cage (Figure 3). The user who operates the PHANTOM Omni should be able to blindly find the opened quarter of the cage guided by the force haptic feedback to the Omni.

\section{RESULTS AND DISCUSSION}

The FE model with adjusted material parameters (from inverse FE analyses) could predict the maximum radial deformation with an acceptable agreement with the experimentally measured deformations. Figure 4 and Table I. present the deformation in both FE model and experiment for all four weights.

The calibration experiments revealed high sensitivity of voltage changes to the force orientation, as illustrated in Figure 5-a for a representative strain gauge. As expected, when larger weights were applied, the voltage amplitude increased (Figure 5-a). The calculated strains in the FE models, at the nodes where the strain gauges were attached, showed similar trend to the measured voltage, as presented for a representative strain gauge in Figure 5-b.

The four strain gauges showed relatively similar behavior when the orientation of the weight changed (Figure 6-a). The (absolute) voltage at each strain gauge was measured to be maximum when the load directly applied to each strain gauge corresponding angle (SG1: $0^{\circ}, \mathrm{SG} 2: 270^{\circ}, \mathrm{SG} 3: 180^{\circ}$ and SG4: $\left.90^{\circ}\right)$.

The changes in measured voltage while changing the weight orientation as indications for strain changes, were confirmed by FE outcomes (Figure 6-b). each strain gauge experienced the peak strain when the weight was applied to its corresponding angle. Interestingly, the strain at the strain gauges were only negative when the loads were applied at the either next points $\left( \pm 22.5^{\circ}\right)$.

TABLE I. MAXIMUM DEFORMATION MEASURED EXPERIMENTALLY VERSUS PREDICTED BY FE MODEL FOR DIFFERENT EXTERNAL WEIGHTS

\begin{tabular}{|c|c|c|}
\hline \multirow{2}{*}{ Weight (gr) } & \multicolumn{2}{|c|}{ Maximum Deformation (mm) } \\
\cline { 2 - 3 } & Experiment & Finite Element Simulation \\
\hline 62.6 & 4.0 & 3.7 \\
\hline 80.6 & 5.7 & 5.1 \\
\hline 112.8 & 9.9 & 9.1 \\
\hline 167.5 & 13.1 & 14.7 \\
\hline
\end{tabular}

Although the strain gauges attached to the sensing module showed relatively symmetrical measurements, the voltage amplitude varied in different strain gauges. This can be due to the differences in attachment of the strain gauges in terms of variations in gauges orientation and attachment itself (gluing). Besides, initial deformation of the gauges caused during attachment process can also induce different measurements. In our set-up, as all the strain gauges were calibrated separately, this could not lead to under-estimation or over-estimation of the force.

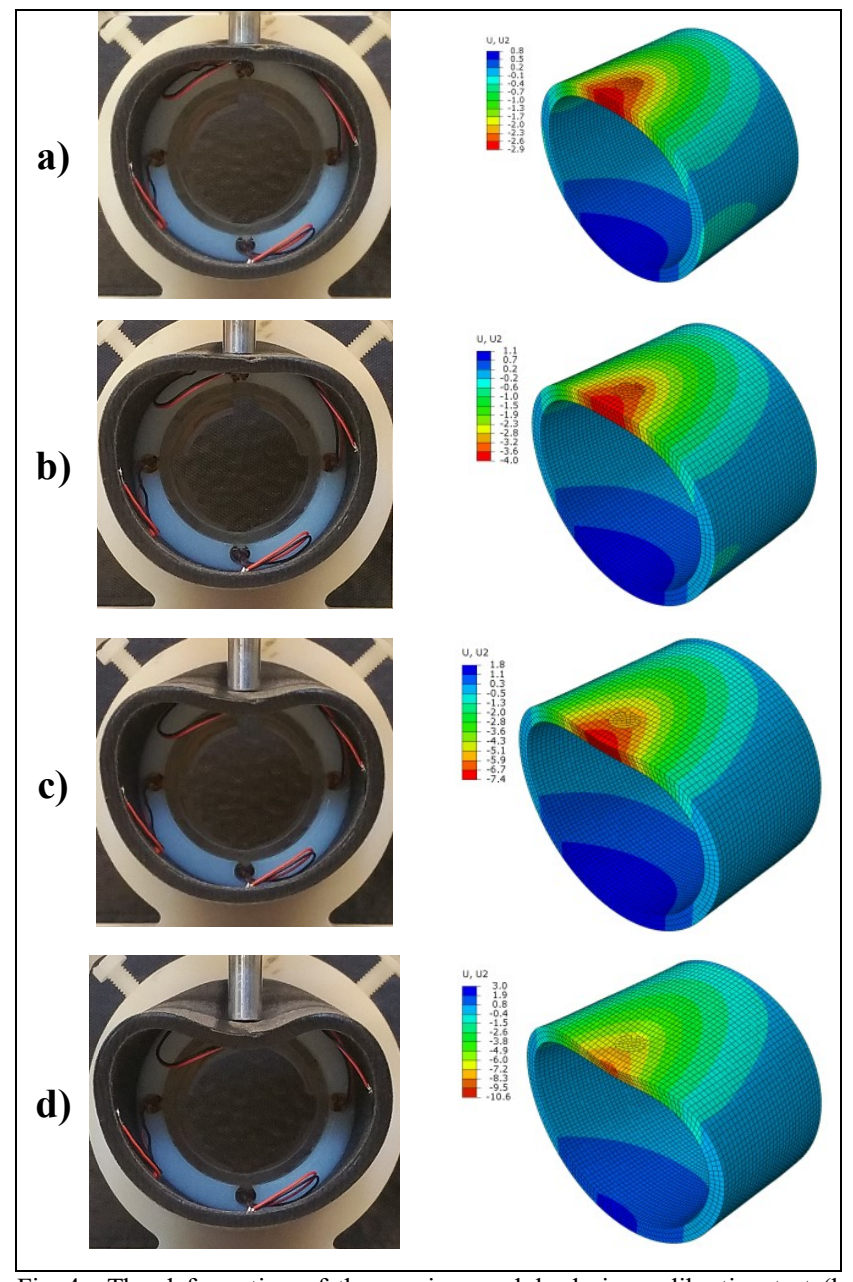

Fig. 4. The deformation of the sensing module during calibration test (left column) and simulated by FE model (right column), for the external weight of 62.6 gr (a), 80.6 gr (b), 112.8 gr (c), and 167.5 gr (d).

In validation experiment, using the developed haptic feedback system, the user could blindly be guided to the opening of the cage. As future works, the sensing module needs to be further optimized using finite element optimization routines in terms of material durability. It also needs to be down sized to the realistic dimensions of the organs of application. A more complicated experiment (e.g. cadaveric experiment) can give a better insight into the realistic interactions with tissues.

\section{CONCLUSION}

In conclusion, in the current study, a flexible sensing module with low risk of tissue damage was developed to determine the amplitude and direction of the external forces. Subsequently, a haptic feedback system was developed to control the directional bending of a soft robotics endoscopic segment. Finite element analysis was implemented for a better understanding of the sensing module mechanical behavior and can be a beneficial tool for further improvements of the sensing modules. The developed force controlled haptic feedback system enhanced the operator navigation. 


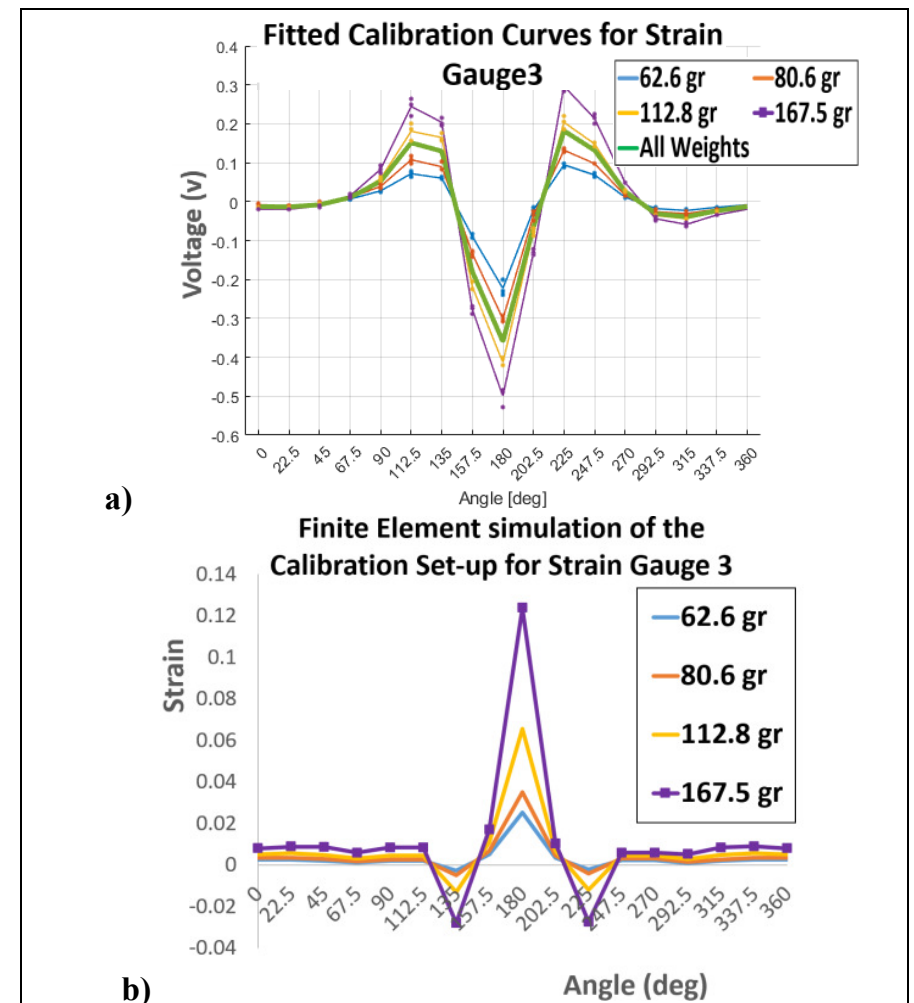

Fig. 5. The voltage meaured by one of the strain gauges (SG3) while different weights were applied to the sensing module in different orientations (a), and the strain at the same strain gauge calculated by FE model (b)

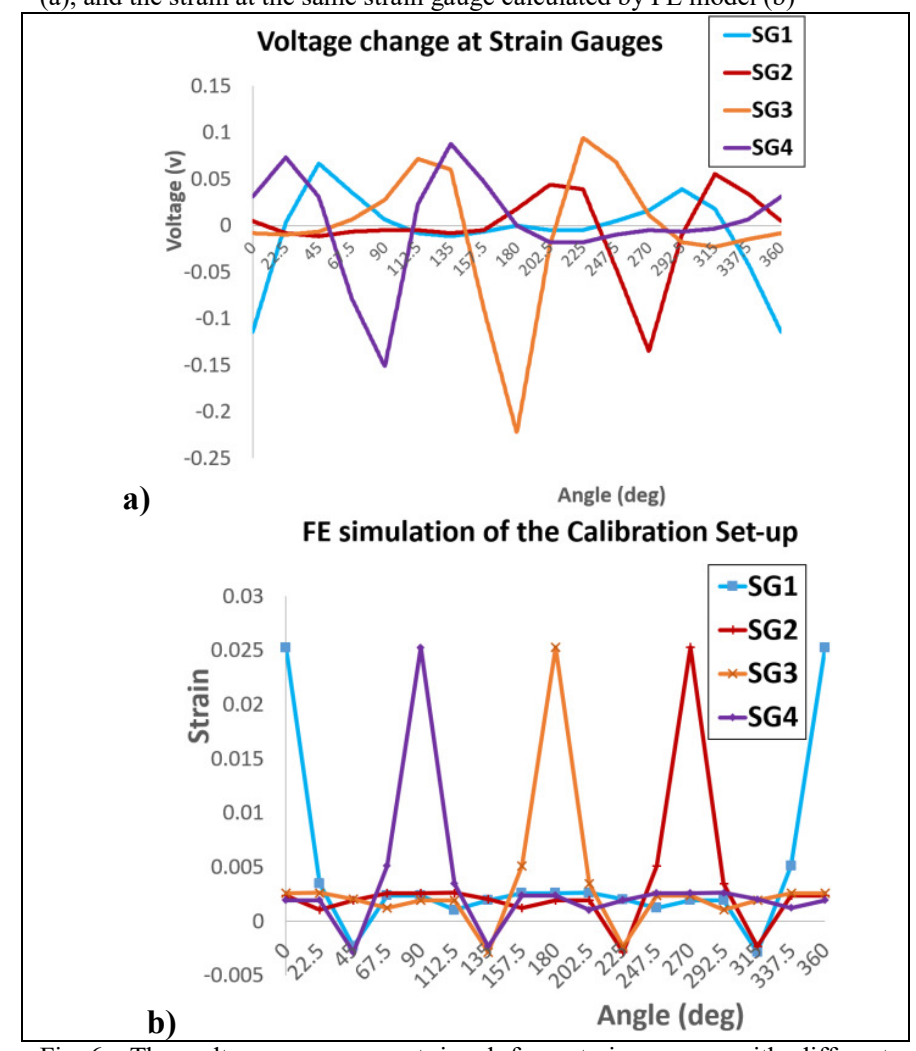

Fig. 6. The voltage measurement in al four strain gauges with different orientational configuration of the sensing module while the 62.6-gr weigth was applied (a), and the strain calculated by FE model at four strain gauges with a similar loading condition to the experiment (b).

\section{ACKNOWLEDGMENT}

The Authors would like to thank Technical Medical (TechMed) Centre, University of Twente, for their financial supports to the Soft Robotics project. We also thank Hendrik Kuipers for his technical support.

\section{REFERENCES}

[1] O. A. J. Van Der Meijden and M. P. Schijven, "The value of haptic feedback in conventional and robotassisted minimal invasive surgery and virtual reality training: A current review," Surg. Endosc. Other Interv. Tech., vol. 23, no. 6, pp. 1180-1190, 2009.

[2] M. Mura et al., "Vision-based haptic feedback for capsule endoscopy navigation: a proof of concept," $J$ Micro-Bio Robot., vol. 11, no. 1-4, pp. 35-45, Jun. 2016.

[3] R. Reilink, S. Stramigioli, A. M. L. Kappers, and S. Misra, "Evaluation of flexible endoscope steering using haptic guidance," Int. J. Med. Robot. Comput. Assist. Surg., vol. 7, no. 2, pp. 178-186, Jun. 2011.

[4] H. Yamanaka et al., "Measurement of the Physical Properties during Laparoscopic Surgery Performed on Pigs by Using Forceps with Pressure Sensors.," $A d v$. Urol., vol. 2015, p. 495308, 2015.

[5] D. Smit, E. Spruit, J. Dankelman, G. Tuijthof, J. Hamming, and $\mathrm{T}$. Horeman, "Improving training of laparoscopic tissue manipulation skills using various visual force feedback types.," Surg. Endosc., vol. 31, no. 1, pp. 299-308, 2017.

[6] M. N. Appleyard, C. A. Mosse, T. N. Mills, G. D. Bell, F. D. Castillo, and C. P. Swain, "The measurement of forces exerted during colonoscopy," Gastrointest. Endosc., vol. 52, no. 2, pp. 237-240, Aug. 2000.

[7] B. Po, M. Yeung, and T. Gourlay, "A technical review of flexible endoscopic multitasking platforms," Int. J. Surg., vol. 10, pp. 345-354, 2012. 